\title{
Sharing Ancient Wisdoms across the Semantic Web using TEI and ontologies
}

\author{
Charlotte Tupman, Department of Digital Humanities, King's College London
}

Anna Jordanous, Centre for e-Research, King's College London

\section{Introduction}

This paper explores the approach of the Sharing Ancient Wisdoms (SAWS) ${ }^{1}$ project to the publication and analysis of the tradition of wisdom literatures in ancient Greek, Arabic, Spanish, and other languages. The SAWS project edits and presents the texts digitally, in a manner that enables linking and comparisons within and between anthologies, their source texts, and the texts that draw upon them (referred to here as 'recipient texts'). It is also creating a framework through which other projects can link their own materials to these texts through the Semantic Web, thus providing a focal point for the development of scholarship on these texts and their related manuscripts. The project is funded by HERA (Humanities in the European Research Area) as part of their programme to investigate cultural dynamics in Europe, and constitutes teams at the Department of Digital Humanities and the Centre for e-Research at King's College London, the Newman Institute Uppsala in Sweden, and the University of Vienna.

The wisdom literatures included in the SAWS project are a feature of the broader tendency in antiquity and the Middle Ages to take extracts from larger texts containing wise or useful sayings, and to circulate these anthologies widely. This was done in order to address the problem of the cost and rarity of full, original texts, and was a key method by which ideas and morals were circulated across different countries and languages (Rodríguez Adrados 2009, p. 91-97 on Greek models; Gutas 1981). SAWS focuses on gnomologia (or florilegia)manuscripts that collected moral or social advice and philosophical ideas-although its methods and tools are applicable to other manuscript types (such as medieval scientific or medical texts); the ability to extend our methodology beyond gnomologia is an important feature of the project (Richard 1962, cols. 475-512).

When new gnomologia were created, they tended to be composed of smaller extracts of earlier works, rather than simple copies. The sayings were selected from various manuscripts, reorganised or reordered, words or sentence structures modified, and sometimes an entire saying or part of a saying was attributed to a different author or philosopher from that of the source text. These texts were frequently translated into other languages (such as from Greek into Arabic), and again these tended to be variations rather than simple translations. Such collections were eventually translated into western European languages-in fact, the first book ever published in England (Caxton 1477) was one such collection. This body of texts, therefore, can be regarded as a complex network or graph of manuscripts and individual sayings that are interrelated in a great variety of ways. An analysis of these relationships can reveal much about the dynamics of the cultures that created and used these texts. Here we describe how we digitally encode relationships between texts using TEI markup and RDF. We discuss how our representational choices afford new ways of exploring and understanding the texts and their inter-relationships, through Semantic Web tools.

\section{Identifying and representing digitally the relationships between texts}

The recording and visualisation of the links within and between the gnomologia, between these collections and their source texts (e.g., Aristotle's writings), and between these collections and

\footnotetext{
${ }^{1}$ http://www.ancientwisdoms.ac.uk/
} 
their recipient texts (e.g. the 11th-century Strategikon of Kekaumenos) is a task that must be approached systematically. Crucially, we should do this in a way that can be replicated by others (either using their own tools or ours), so that our collection of texts can act as a starting point for an expandable corpus that takes this approach far beyond our own project.

Before the methodology is explained, we should look at an example of the nature of the texts under consideration. This saying is from Gnomologium Vaticanum (no. 87):

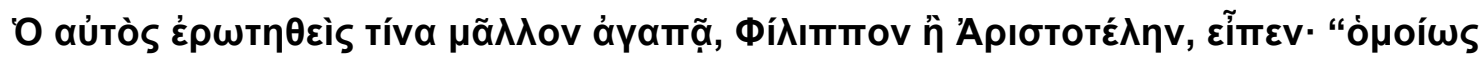

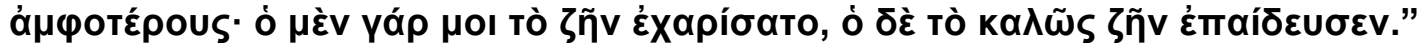

Alexander, asked whom he loved more, Philip or Aristotle, said: "Both equally, for one gave me the gift of life, the other taught me to live the virtuous life."

We can identify that this saying, or section of text, exists in various forms in earlier works, and that there are relationships that can be defined between our saying above and those quoted below (and indeed between the various examples given below):

Plutarch, Life of Alexander 8.4.1:

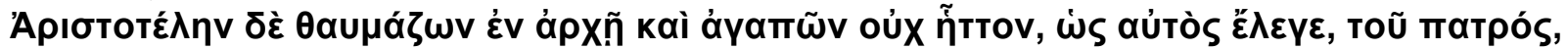

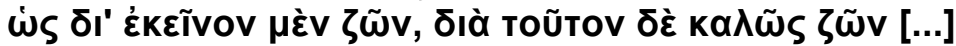

Alexander admired Aristotle at the start and loved him no less, as he himself said, than his own father, since he had life through his father but the virtuous life through Aristotle [...]

Diogenes Laertius, Life of Aristotle 5.19:

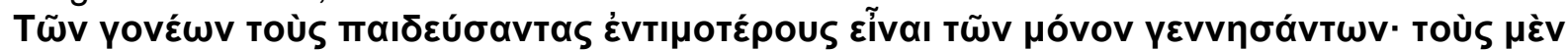

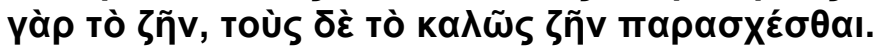

Aristotle said that educators are more to be honored than mere begetters, for the latter offer life but the former offer the good life.

Pythagorus? Selections from the Sayings of the Four Philosophers: (B) Pythagoras saying 18 (ed. Gutas):

ع دم سبب صلاح الحياقوقال البآباء دم سبب الحياة والحكوما

He said: Fathers are the cause of life, but philosophers are the cause of the good life.

In this final example, we can see that the saying has actually been attributed to a different author (Pythagoras), rather than Aristotle or his pupil Alexander. Alternative attributions are a common feature of these kinds of texts, and they add yet another layer of complexity to the types of relationships that can be discovered and described.

We need, therefore, to find suitable methods for:

1) Inserting links between sections of text (both within and between documents, some of which exist in digital form, and some of which do not);

2) Providing a means by which scholarly assertions defining the nature of these relationships can be shared and recorded in a systematic manner; this is particularly important in enabling individual scholars to take credit for (and indeed responsibility for) the assertions that they make in identifying links and defining the nature of those relationships.

\section{The basic unit of interest}


We must therefore define, first of all, the basic unit of intellectual interest to us (a 'section' or 'segment' of text), which is the saying (or part of the saying). In order to encode these units, SAWS has chosen to use the Text Encoding Initiative (TEI) Guidelines, which provide guidance as to how to use XML to 'mark up' (i.e., encode) texts of various types. ${ }^{2}$ Taking the TEI manuscript schema as a basis, the SAWS team at King's College London has designed its own schema which can be used specifically for the encoding of gnomologia and their related texts. The use of a schema ensures that there is consistency in the markup that we use to encode our texts: as part of the project, the SAWS schema will be made freely available to all scholars who wish to use it for their own purposes.

SAWS uses the <seg > (arbitrary segment) element to define the basic unit of intellectual interest (such as a saying [statement] together with its surrounding story [narrative]). For example:

<seg>Alexander, asked whom he loved more, Philip or Aristotle, said: "Both equally, for one gave me the gift of life, the other taught me to live the virtuous life".</seg>

This saying contains both a statement and a narrative, so each of these are therefore marked up in their own <seg > element, using attributes to indicate the nature of the segment ("contentltem" is simply a term that the SAWS project uses to define the whole saying):

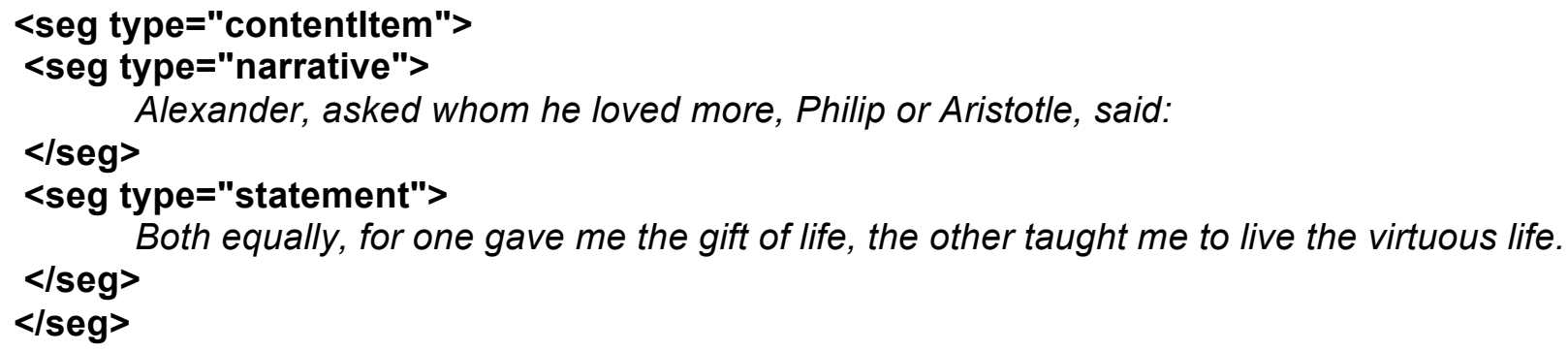

In order to differentiate one <seg> from another, each <seg> element is given an @xml:id attribute whose value is a unique identifier (which is automatically generated using simple XSLT: this does not need to be done by hand). The identifier differentiates one $<\mathbf{s e g}>$ from all other examples of <seg>, for instance:

NB The project ultimately decided to adopt the <seg type="statement" xml:id="K.al-Haraka_ci_s1"> Canonical Text Services (CTS) notation, developed by the Homer Multitext Project for identifying and citing texts and parts of texts: «http://

("K.al-Haraka_ci_s1" is the unique identifier). www.homermultitext.org/hmt-doc/cite/texts/ ctsoverview.htmls

This method allows each intellectually interesting unit (as defined by the scholar who is marking up the text) to be distinguished from each other unit, thus providing a means of referring directly to a specific, often very brief, section of the text.

\section{Describing textual relationships with an ontology}

Next, we must have a systematic way of defining the relationship between one section of text and another. The use of a systematic method is important for two reasons: to ensure consistency in the descriptive terms that we use across the project, and, crucially, to develop a shared vocabulary between SAWS and other projects to which we wish to establish links (and

\footnotetext{
${ }^{2}$ http://www.tei-c.org/index.xml
} 
which wish to link their data to ours). We have therefore taken every possible opportunity to explore with other scholars the terms they would use to describe the relationships that they observe within, and between, their texts. Relationships identified include terms such as isCloseRenderingOf, isLooseTranslationOf, isVerbatimOf, and a variety of other terms that represent in an agreed form the different ways in which sections of text are connected to one another. These are stored in the form of an ontology, which lists and gives details of all the types of relationship that have been defined. As ontologies are easily extensible, any relationships of interest identified in the future can also be included: again, the SAWS methodology has been designed to enable its use and modification by others.

We are representing these relationships using an ontology that extends the FRBR-oo model (Doerr and LeBoeuf 2007), which is the harmonisation of the FRBR model of bibliographic records (Tillett 2004) and the CIDOC Conceptual Reference Model (CIDOC-CRM; Doerr 2003). The SAWS ontology, ${ }^{3}$ developed through collaboration between domain experts and technical observers, models the classes and links in the SAWS manuscripts. Basing the SAWS ontology around FRBR-oo allows us to reuse much existing vocabulary for both the bibliographic (FRBR) and cultural heritage (CIDOC) aspects being modelled.

\section{Adding relationships to the TEI document}

Using this underlying ontology as a basis, relationships between (or within) texts can be added to the TEI documents using the TEI element <relation/>. Three entities must be represented: the subject being linked from, the object being linked to, and a description of the link between them. These three entities constitute RDF (Resource Description Framework) 'triples'. The subject entity is represented by the @xml:id of the section of text that is being linked from; the object entity is represented by the @xml:id of the section of text that is being linked to; and the third entity-the nature of the link itself-is represented by another attribute, @ref, the value of which is drawn directly from the ontology in which we store all the relationships.

In order to insert this information into the TEl document, we use the element <relation>, which has recently been adopted by the TEl community for the purpose of placing RDF markup in TEI documents. When used for this purpose, <relation> takes three main attributes (i.e. the RDF triples), and a fourth that we considered important to include:

- @active - the @xml:id of the subject being linked from;

- @passive - the @xml:id or URI (Uniform Resource Identifier) of the object being linked to;

- @ref - the description of the relationship, which is drawn directly from the list of relationships in the ontology;

- @resp - (responsibility): the name or identifier of a particular individual or resource, such as a bibliographic reference. This enables the recording of the identity of the person(s) responsible for making the assertion about the existence of a relationship between two sections of text. We felt it was important to include this facility, both from the point of view of ensuring proper credit for those who make scholarly assertions about the texts, and also as a means of being able to trace decision-making more easily.

Here is an example of a link that has been identified by one of our teams between two small sections of text in Arabic and Greek manuscripts:

\footnotetext{
${ }^{3}$ http://purl.org/saws/ontology
} 


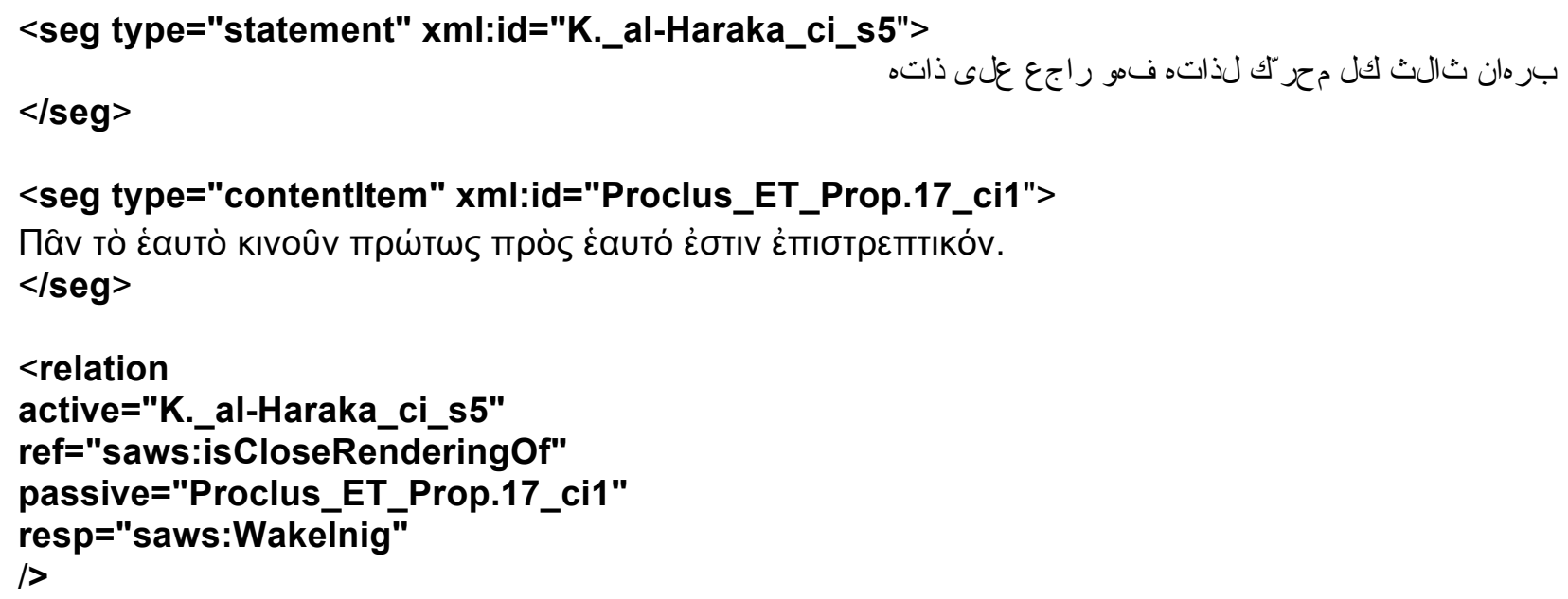

This is equivalent to stating that the Arabic segment identified as "K._al-Haraka_ci_s5" is a close rendering of the Greek segment identified as "Proclus_ET_Prop.17_ci1", and that this relationship has been asserted by Elvira Wakelnig (this could instead be a pointer to a bibliographic reference, if desired).

The definition of 'isCloseRenderingOf' has been agreed upon and documented within the ontology, and the schema has been populated from the ontology so that a drop-down menu appears in the XML editor, from which the required value of @ref can be selected. The $<$ relation> element can be placed anywhere within the TEI document, or indeed in a separate document if required. Several different coding strategies have emerged in our own annotations for SAWS because of personal preferences for different markup approaches, with the most popular strategies being to place each individual <relation> element immediately after the closing tag of the <seg $>$ identified as the active entity, or to include at the end of a TEI file a group of all <relation> statements relevant to a particular document. Either method is equally appropriate.

There are clear benefits in linking to external sources where possible, as well as including links within the documents themselves, thereby enhancing the semantic content of our texts by viewing them in the context of other relevant information that is stored externally to our documents. Linking our texts to external sources also provides an alternative point of entry to our texts. For this purpose, the SAWS project also marks up our texts with semantic links to collections of data on the ancient world where possible, such as the Pleiades historical gazetteer of ancient places, ${ }^{4}$ and the Prosopography of the Byzantine World, ${ }^{5}$ which aims to document all the individuals mentioned in textual Byzantine sources from the seventh to thirteenth centuries. These links will be supplemented with links to the DBpedia dataset and to the geonames.org modern geographical dataset. ${ }^{6}$ Collaborative discussions are also currently underway to identify how we can best mark up links to existing relevant documents such as those stored in the Perseus Digital Library, ${ }^{7}$ which holds editions of some of the texts that have been identified as source texts for the gnomologia.

\section{Dealing with editorial uncertainty}

\footnotetext{
${ }^{4}$ http://pleiades.stoa.org/

${ }^{5}$ http://www.pbw.kcl.ac.uk/

${ }^{6}$ Geonames: http://www.geonames.org/ , DBpedia: http://dbpedia.org/

${ }^{7}$ http://www.perseus.tufts.edu/
} 
The relations being expressed about our texts are often subjective; it may be that people will have different opinions about whether or not the assertion of a particular relationship is validfor example, whether or not one text is sourced from another, or which relationship is most appropriate to use in any particular case. Also, it may be that individual editors see the potential existence of a link between two texts, but are not completely certain about the nature of that link.

The set of link types provided in the SAWS ontology have been developed with the specific vocabulary requirements of our textual scholars in mind, in order to describe relationships using the appropriate terms. Furthermore, explanatory definitions are provided for all terms in the ontology, ${ }^{8}$ to assist editors in choosing the appropriate relationship.

In a scenario in which editors disagree on what relationship description is best to use, or where there is disagreement on the validity of asserting a relationship at all, it is vital to be able to record the identity of the individual making the assertion (using the @resp attribute in TEl and reification ${ }^{9}$ in RDF). As well as crediting scholarly assertions to the appropriate editor, @resp also allows conflicting opinions to be asserted alongside each other without needing to make an artificial choice of one opinion over the other. For example, if we have a damaged manuscript in which some of the text is lost or faded, one editor may think it most likely that this manuscript is a verbatim copy of another manuscript, and so asserts this. Another editor, on the other hand, may disagree, believing this manuscript to be only a close rendering of the other manuscript, and thus can assert this under his own responsibility.

We are currently exploring the best way to handle editorial uncertainty, for instance in cases in which the individual making an assertion about a relationship cannot be completely certain that their assertion is correct due to insufficient corroboratory evidence, or similar reasons. With source material such as ours, and the complexity of the relationships that are being identified, it is inevitable that cases like this will arise. We would like to be able to express these types of uncertainty in a manner that allows the reader to see immediately where a relationship is being identified tentatively, rather than with a high degree of certainty.

There are two main ways in which we might express editorial uncertainty in the markup. One option would be to add a certainty attribute (@cert) to the <relation> element. This would be the simplest method, a particularly relevant concern for editors with less technical proficiency or confidence, and would enable the editor to include the expression of certainty within the same element as the rest of the information about that relationship. For instance:

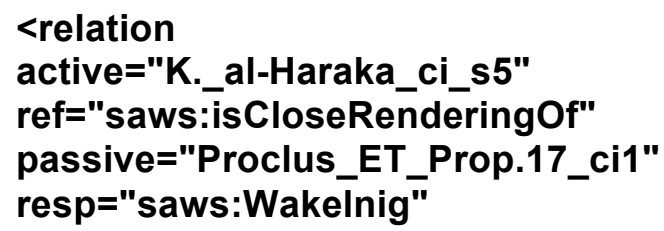

\footnotetext{
${ }^{8}$ Definitions can be viewed directly in the OWL ontology at http://purl.org/saws/ontology and also through ontology display and documentation tools such as the LODE tool (http://www.essepuntato.it/lode) at http://www.essepuntato.it/lode/owlapi/reasoner/http://purl.org/saws/ontology .

9 In RDF, a reified statement is a statement of a subject-predicate-object triple that incorporates higherorder statements about the triple itself, such as who is making the assertion represented by the triple. Reification therefore allows us to represent the @resp attribute of a relationship within the RDF.
} 
cert="low"

1>

However, it could be argued that using this method does not make it sufficiently clear what we are ascribing a low certainty to: for instance, does the certainty refer to the existence of a relationship, or to the type of relationship, or to the identity of the passive text?

If we wish to be more explicit about what the expression of uncertainty refers to, we can instead use a <certainty> element, and point this to the specific part of the <relation> that we are describing. So, for instance, if we would like to say that we are uncertain about whether or not a relationship actually exists at all, we can express this in the markup in the following way:

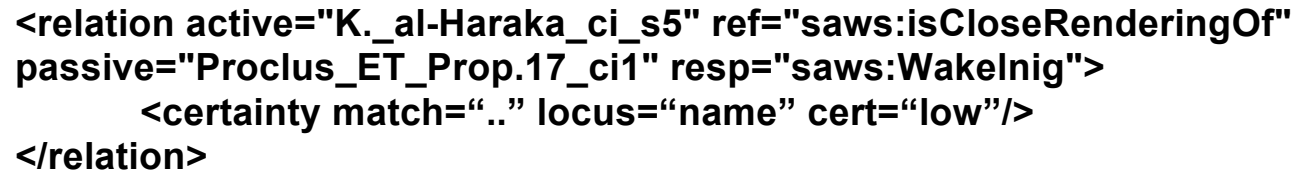

Note that here we are placing <certainty $>$ within <relation> (thus it is no longer an empty element), and we are using XPath to match the <certainty> to its parent element (expressed with the two full stops). The @locus allows us to describe what we are not certain about, viz., the name of the element (in other words, we are not entirely certain whether or not a relationship exists here).

If we wish to express uncertainty about one of the attributes within <relation>, for instance the type of relationship that exists, we can use a similar method, but point to a specific attribute rather than to the element name:

<relation active="K._al-Haraka_ci_s5" ref="saws:isCloseRenderingOf" passive="Proclus_ET_Prop.17_ci1" resp="saws:Wakelnig"> $</$ relation $>$ <certainty match="../@ref" locus="value" cert="Iow"/>

In this example, the @match refers directly to the parent element's @ref, and the @locus expresses that we are uncertain about the value of that attribute-in other words, we are uncertain about the nature of the relationship that has been identified. Similarly we could apply this method to any of the attributes within <relation $>$.

It is possible to express degrees of certainty using @degree, but at this stage we feel that this would add an unnecessary layer of complexity to the markup. It is, however, something that might be worth exploring in a future stage of the project if it was found to be a useful method of making the editorial process more explicit.

\section{Justifying our choice of representation of RDF in TEI documents}

As described above, we are using RDF triples $^{10}$ to mark up information of semantic interest such as relations between the text and links to external entities. Thus our relations can be published as Linked Data on the Semantic Web, where they can be shared, browsed through,

\footnotetext{
${ }^{10}$ http://www.w3.org/RDF/
} 
searched and queried. Particularly for our purposes (and those of others) concerning TEI XML documents, which are a popular product of digital humanities research, we prefer that RDF annotations should be added to XML documents without extensive changes being required in either the variant of XML being used for the source document, or in the skills and workflow being used in the markup process. This last point is of particular concern for less-technical users who are inexperienced with markup. Keeping structural, syntactical, and semantic information in the same documents where possible also makes the process of markup more simple and less error-prone for non-technical users who wish to mark up documents with their annotations, though it is acknowledged that this is not always possible. To date, no method for accommodating TEI and RDF in the same document has been adopted as standard by the TEl community, though several approaches have recently been offered.

It is desirable (e.g., for SAWS) to be able to mark up triple-like relations directly in TEI, particularly if those relations are specific to the subject domain of the original text and/or if the relations indicate semantic information which cannot currently be encoded using TEI markup. The <relation> element that we have chosen to use in the SAWS project has recently been recommended by the TEI for encoding RDF relations in a TEI document, representing the Subject-Predicate-Object triple format through the values of the attributes of <relation> as described above.

This allows TEI to express more semantic information, through the encoding of RDF triples, without requiring major changes within TEI. Furthermore, RDF can be included directly in TEI markup, allowing researchers to use the workflow and tools they are already accustomed to rather than introducing a requirement for new tools to be learnt and used, external to the existing workflow. This is particularly helpful for users of TEI who do not have a strong technical background.

\section{The benefits of RDF for information exploration and retrieval}

Once information is available in RDF format, it can be queried and reasoned with, leading to the potential generation of new information from reasoning logically with the existing information. We have already seen at least one example within SAWS in which this approach has been helpful. Two of our Arabic texts were linked to one translation but were not linked to each other (in other words, the scholar had made an assertion about the link between each text and the translation, but had not yet asserted a link between the two Arabic texts), but we were able to highlight the links between the Arabic texts by navigating links between the two documents via the translation. New links such as this can be automatically discovered and added to our existing links using Semantic Web tools.

This approach also allows information to be derived from external data sources that are referenced by RDF triples. To illustrate this in the use case of SAWS, information in the Pleiades historical gazetteer can be consulted when constructing queries. Researchers can ask to see, for example, all texts that refer to a particular geographical location, even if they use different place-names to refer to that geographical location (for instance, cases in which places were referred to by different names in different historical periods). For SAWS, this assists us with the frequent issue of manuscripts being in different languages with different character sets (compare for example Ancient Greek and Arabic). By examining the place-names mentioned in the SAWS manuscripts in the context of the information in the Pleiades ontology, we have a precise geographical reference for each place. 
For example the place "Aphrodisias" (URI http://pleiades.stoa.org/places/638753) was known by the names:

- Nivón (in the Classical period),

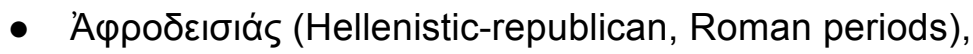

- $\Lambda \varepsilon \lambda \dot{\varepsilon} \gamma \omega \mathrm{V}$ mó̀ıs (unspecified period),

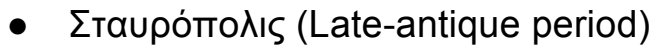

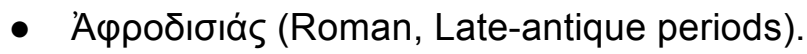

Developing this example, we can disambiguate between Aphrodisias located in modern-day Turkey and the Aphrodisias located by modern-day Spain (URI http://pleiades.stoa.org/places/255978/), between which the textual information in the TEI alone would not allow us to distinguish.

It is possible that in some cases, the editor might not be able to say whether a placename refers to one particular location or to another; in this case, the editor could express this by including both possibilities (in other words, including more than one Pleiades reference) and then add an expression of uncertainty to both locations, to indicate that each referenced place could possibly be the place that is mentioned in the text, but that the editor is not sure enough to make either of the assertions with a high degree of certainty. If necessary, an indication of the degree of certainty that the editor has in any particular place-name identification can be added, for instance in cases in which an editor feels that one particular location is more likely to be the place mentioned in the text.

Returning to the issues of the SAWS manuscripts being written in various languages (Ancient Greek and Arabic being the two main languages, and some related documents in Spanish, Latin, and English, to date): although the TEl documents contain transcriptions of manuscripts in the original language, the use of RDF and linking allows the manuscript information to transcend linguistic boundaries to some extent, as parts of the text can be linked to resources which are more language-neutral (e.g., the person 'Aristotle' can be represented by the URI http://dbpedia.org/resource/Aristotle independently of whether he is referred to as Aristotle, Арıбтотغ́^ns, أرسطو , Aristoteles, Aristóteles or other alternative forms in the original document). This is particularly helpful in studying the transmission of information in the manuscripts across languages, especially if the researcher does not have sufficient skills to navigate between the different languages.

\section{Future work}

Having established our methodology and annotated a selection of texts with TEI and RDF for demonstration purposes, the research process of linking our texts and annotating relationships is now continuing in earnest. Currently we have approximately twenty texts marked up in TEI $\mathrm{XML}$, with more texts in preparation as the project grows and extends to collaborators. Relationships are being added to these texts on an active and ongoing basis, as the scholars record their knowledge and opinions on how the texts interlink and link to external entities. We are also actively working with existing and potential collaborators-discussing how the ontology suits the needs of other scholars-to establish good practice for linking with other text 
repositories and to provide training to those who are seeking help in marking up their texts using the SAWS approach. ${ }^{11}$

In terms of publishing our texts as digital editions, we have a functional demonstration version of the online environment in which texts and their relationships will be published. This version is undergoing further development in response to post-demonstration feedback from scholars, prior to becoming publicly accessible online at http://www.ancientwisdoms.ac.uk. We are also exploring alternative ways in which the relationships can be visualised, searched, and analysed, in order to give scholars new visual perspectives on these links.

\footnotetext{
${ }^{11}$ One of the key outcomes for the SAWS project of the Methods and means for digital analysis of ancient and medieval texts and manuscripts workshop was the connection that we made with the project of Samuel Rubenson and his colleagues at Lund. They are approaching the same research questions using a different methodology, and following our meeting in Leuven we have organised a workshop to discuss our respective projects and to discover how best we can work together towards achieving our shared aims. 
Bibliography

F. Rodríguez Adrados (2009), Greek Wisdom Literature and the Middle Ages: the Lost Greek Models and their Arabic and Castilian Translations, trans. J. Greer, Bern; New York: Peter Lang, p. 91-97.

W. Caxton (1477), The Dictes and Wise Sayings of the Philosophers, London: Elliot Stock (repr. 1877).

M. Doerr (2003), 'The CIDOC CRM - an Ontological Approach to Semantic Interoperability of Metadata', in Al Magazine, 24, p. 75-92.

M. Doerr and P. LeBoeuf (2007), 'Modelling Intellectual Processes: The FRBR - CRM Harmonization', in C. Thanos, F. Borri, and L. Candela (eds.), Digital Libraries: Research and Development, Berlin; Heidelberg: Springer (Lecture Notes in Computer Science, 4877) p. 114123.

D. Gutas (1981), 'Classical Arabic Wisdom Literature: Nature and Scope', in Journal of the American Oriental Society, 101, p. 49-86.

M. Richard (1962), 'Florilèges grecs', in Dictionnaire de Spiritualité, 5, cols. 475-512.

B. Tillett (2004), 'What is FRBR? a conceptual model for the bibliographic universe', in Library of Congress Cataloging Distribution Service, 25, p.1-8. 